\section{Photoacoustic lifetime imaging of dissolved oxygen using methylene blue}

\author{
Shai Ashkenazi \\ University of Minnesota, Department of Biomedical \\ Engineering, 312 Church Street Southeast, Minneapolis, \\ Minnesota 55455
}

\begin{abstract}
Measuring distribution of dissolved oxygen in biological tissue is of prime interest for cancer diagnosis, prognosis, and therapy optimization. Tumor hypoxia indicates poor prognosis and resistance to radiotherapy. Despite its major clinical significance, no current imaging modality provides direct imaging of tissue oxygen. We present preliminary results demonstrating the potential of photoacoustic lifetime imaging (PALI) for noninvasive, 3-D imaging of tissue oxygen. The technique is based on photoacoustic probing of the excited state lifetime of methylene blue $(\mathrm{MB})$ dye. $\mathrm{MB}$ is an FDA-approved water soluble dye with a peak absorption at $660 \mathrm{~nm}$. A double pulse laser system (pump probe) is used to excite the dye and probe its transient absorption by detecting photoacoustic emission. The relaxation rate of MB depends linearly on oxygen concentration. Our measurements show high photoacoustic signal contrast at a probe wavelength of $810 \mathrm{~nm}$, where the excited state absorption is more than four times higher than the ground state absorption. Imaging of a simple phantom is demonstrated. We conclude by discussing possible implementations of the technique in clinical settings and combining it with photodynamic therapy (PDT) for real-time therapy monitoring. (c) 2010 Society of Photo-Optical Instrumentation Engineers. [DOI: $10.1117 / 1.3465548]$
\end{abstract}

Keywords: hypoxia; photoacoustic imaging; triplet lifetime; oxygen quenching; methylene blue; photodynamic therapy.

Paper 10183LR received Apr. 8, 2010; revised manuscript received May 23, 2010; accepted for publication Jun. 23, 2010; published online Jul. 21, 2010.

The balance of oxygen delivery and consumption in tissue is altered by disease processes resulting in change of tissue $\mathrm{O}_{2}$ level $\left(\mathrm{pO}_{2}\right)$ and its spatial distribution. In the case of cancer, for example, tumor growth promotes an increased level of oxygen consumption. The new demand is partially compensated by the formation of new blood vessels (angiogenesis). However, the irregular nature of cancer progression results in large variability in tissue $\mathrm{pO}_{2}$, since tumor regions close to blood vessels are well perfused, while other regions lack oxygen supply and become hypoxic. ${ }^{1,2}$ Because of the important role oxygen plays in cancer biology and tumor progression, imaging of $\mathrm{pO}_{2}$ distribution in tissue can significantly improve cancer screening, cancer diagnosis, therapy planning, and therapy monitoring. This challenge had motivated and

Address all correspondence to: Shai Ashkenazi, Tel: 612-625-6107; Fax: $612-$ 626-6583; E-mail: ashke003@umn.edu driven an extensive effort in developing new tissue oxygen imaging methods. Modalities such as positron emission tomography (PET), ${ }^{3}$ blood oxygen level dependent functional magnetic resonance imaging (BOLD-MRI) ${ }^{4}$ and fluorescence imaging ${ }^{5}$ have been modified to yield $\mathrm{pO}_{2}$-related imaging. However, implementation of these methods in clinical settings is not common due to inherent limitations. BOLD-MRI is only sensitive to blood oxygenation. PET agents indicate activity of cell metabolism, which is only indirectly related to oxygen contents in the tissue. Phosphorescence imaging cannot resolve depth and therefore is not applicable to tissue imaging. There is clearly a strong and unsatisfied clinical need for a tissue oxygen imaging modality.

Recently we proposed photoacoustic lifetime imaging (PALI) as a pump-probe photoacoustic technique for noninvasive, 3-D tissue oxygen imaging. ${ }^{6}$ The technique relies on photoacoustic measurement of the lifetime of an oxygensensitive dye-excited state. A metal core porphyrin dye [Pt(II) octaethylporphine, PtOEP] was successfully used for oxygen sensing. However, with the excitation wavelength in the range of 500 to $540 \mathrm{~nm}$, PtOEP limits penetration depth in tissue to less than $3 \mathrm{~mm}$.

We present here a new implementation of a technique using Methylene blue (MB) as an oxygen sensitive agent. $\mathrm{MB}$ is an FDA-approved, water soluble dye. It has a strong absorption peak at $660 \mathrm{~nm}$ (extinction coefficient is $\left.72,000 \mathrm{~cm}^{-1} / \mathrm{M}\right)$. At this wavelength, tissue optical absorption is relatively low, allowing imaging at a larger penetration depth.

MB has a high quantum yield for triplet state transition and a long triplet lifetime of $79.5 \mu \mathrm{s}$ in a degassed water solution. ${ }^{7}$ In air-saturated aqueous solution, the lifetime drops sharply to less than $2 \mu \mathrm{s}$. The reduced lifetime results from efficient energy transfer to oxygen molecules and formation of singlet oxygen. This makes MB a good photosensitizer for photodynamic therapy (PDT). Its use in PDT had been studied for several clinical applications. ${ }^{8-10}$ The main advantage of $\mathrm{MB}$ as a PDT agent is its efficient topical administration, eliminating skin photosensitivity, one of the side effects of PDT. ${ }^{11}$ The same features make MB suitable for tissue oxygen monitoring by PALI. PALI employs photoacoustic imaging to map tissue optical absorption by detection of an acoustic field emitted by thermoelastic expansion induced by a laser pulse. ${ }^{12}$ In PALI, an excitation pulse of a different wavelength is first applied to excite the dye at a specified time interval before the photoacoustic imaging pulse is fired. This enables photoacoustic probing of tissue absorption at a specified delay after excitation. Repeating it for multiple excitation-probe delay times yields transient absorption data, enabling pixel-by-pixel reconstruction of the excited state lifetime within the imaging volume. In conjunction with oxygen-sensitive dye such as $\mathrm{MB}$, the technique is capable of producing tissue oxygen distribution noninvasively in a $3-\mathrm{D}$ volume. Imaging depth depends mostly on the penetration depth of the excitation light in tissue.

We have designed experiments to test PALI in simple phantom objects containing either one or two dye-filled plastic tubings. The experimental setup used in our measurements is shown in Fig. 1. The excitation pulse is generated by an

1083-3668/2010/15(4)/040501/3/\$25.00 @ 2010 SPIE 


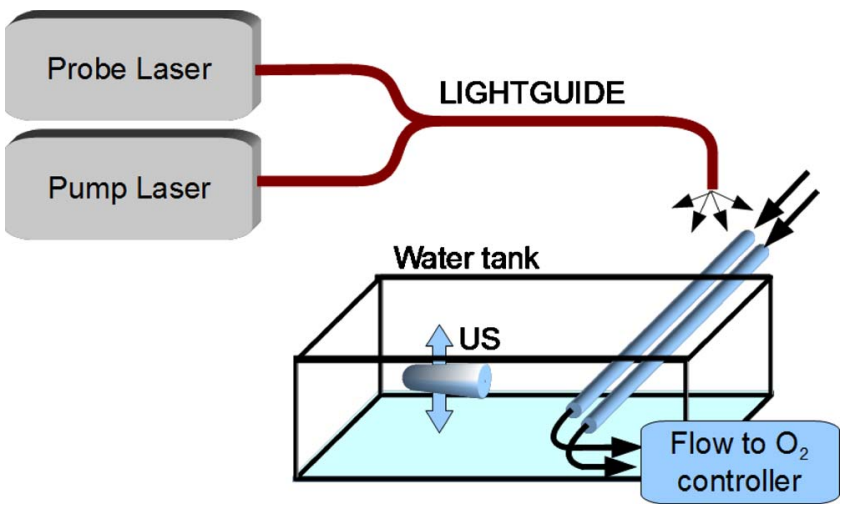

Fig. 1 PALI imaging experimental setup includes a pulsed pump laser $(650 \mathrm{~nm})$, pulsed probe laser $(808 \mathrm{~nm})$, and a light guide that combines both laser outputs and illuminates the phantom object. An ultrasound (US) transducer (10 MHz, focused) mounted on a motorized stage collects the PA signals. The phantom includes two plastic tubes. Two separate closed flow loops control independently the oxygen level dissolved in MB dye solution in each tube. The phantom and ultrasound transducer are immersed in a water tank.

optical parametric oscillator (OPO, Magic prism Opotek, Carlsbad, California) pumped by a tripled NdYAG pulsed laser (Surelite I-10, Coherent, Santa Clara, California). The OPO system allows tunability of the excitation pulse wavelength in the range of 480 to $680 \mathrm{~nm}$. In all experiments described here, the excitation wavelength is $650 \mathrm{~nm}$. A second pulsed-laser OPO system is used for photoacoustic probing (Opotek Rainbow). The second OPO system is tunable in the range of 700 to $960 \mathrm{~nm}$. The relative time delay between pump and probe laser firing is controlled by a programmable logic array (KNJN Saxo FX2 FPGA, California). The output of both OPO systems is coupled to two branches of a Y-branched light guide (Edmund Optics, York, United Kingdom). The common port of the branched light guide is connected to an additional liquid-filled flexible light guide to ensure uniform and equal distribution of the two beams across the illumination aperture. The output from the liquid light guide is directed to illuminate the MB-filled tubing phantom (Tygon, Paris, France, $\mathrm{ID}=0.7 \mathrm{~mm}, \mathrm{OD}=2 \mathrm{~mm}$ ). $\mathrm{MB}$ dye is circulated in the tubing by a peristaltic pump (Masterflex 77300-50, Vernon Hills, Illinois). Oxygen concentration is controlled by bubbling either nitrogen or an air-nitrogen mixture in a cell connected in-line with the flow circulation system. Oxygen level is measured independent of PALI measurements by oxygen electrode (microelectrodes, part 16-732). The phantom is immersed in a water tank, and an ultrasound transducer (GE Panametrics, V311, $10 \mathrm{MHz}, \mathrm{fH=2}$ ) is used for detecting photoacoustic signals. Signals are amplified by an electronic amplifier (JSR Ultrasonics, Pittsford, New York, model DPR300), recorded by a digital oscilloscope (Lecroy Wavejet 354, Chestnut Ridge, New York), and transferred to a computer for processing. A motorized actuator (Zaber Technologies T-LA28, Vancouver, British Columbia) and a linear stage (Thorlabs PT3, Pittsburgh, Pennsylvania) are used for scanning the ultrasound transducer in a direction perpendicular to the tubing's axis.

In a first set of measurements, single tubing was installed. A dye solution of 0.3-mM MB in phosphate buffered saline (PBS) solution $(\mathrm{pH}=7.4)$ was prepared. The solution was

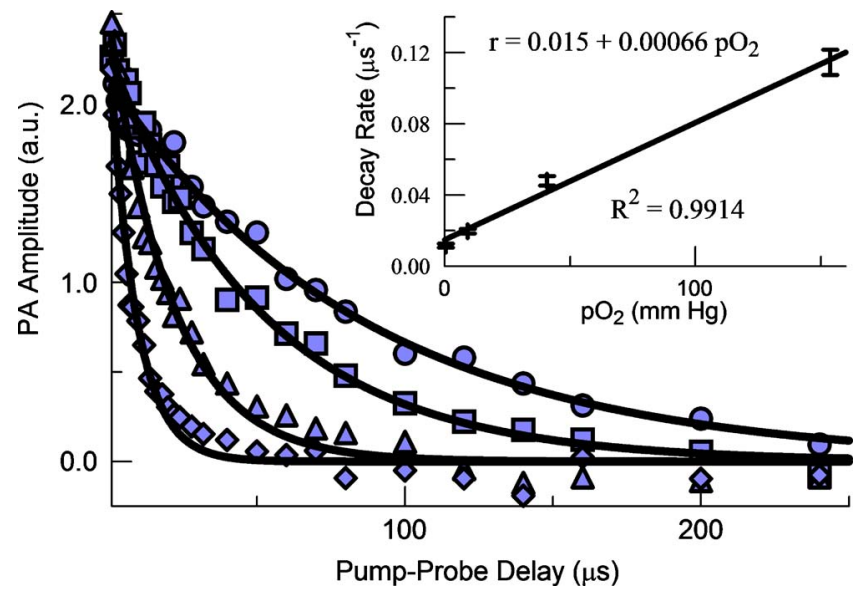

Fig. 2 Photoacoustic signal energy as a function of pump-probe delay time interval acquired at four oxygen partial pressure levels, $0.4 \mathrm{mmHg}(\bigcirc), 8.6 \mathrm{mmHg}(\square), 40 \mathrm{mmHg}(\triangle)$, and $153 \mathrm{mmHg}(\diamond)$. Inset: photoacoustic signal decay rate is plotted against oxygen partial pressure. A linear fit adequately describes the data (coefficient of determination $\mathrm{R}^{2}=0.9914$ ).

then circulated in the tubing system. A constant $\mathrm{pO}_{2}$ was maintained by controlling the ratio of $\mathrm{N}_{2}$ to air-gas bubbling rate in the oxygen control cell. The probe laser system was tuned to $810 \mathrm{~nm}$. The optical fluence at the object location of the pump and probe beams was 1.8 and $2.5 \mathrm{~mJ} / \mathrm{cm}^{2}$, respectively. We have measured the generated photoacoustic (PA) signal at a range of pump-probe time delays. The probe photoacoustic signal was evaluated by first subtracting a signal measured with only the pump laser operating from a signal measured with both the pump and probe lasers on. The signal energy is quantified by integrating the square of the signal amplitude. PA signal energy as a function of pump-probe delay time is given in Fig. 2 for four different $\mathrm{pO}_{2}$ values. A fit-to-exponential decay is performed for each set of measurements. The decay rate (1/lifetime) is plotted as a function of the oxygen level (see Fig. 2 inset). The relationship of decay rate to $\mathrm{pO}_{2}$ is well described by linear dependence.

A basic imaging experiment was performed by installing two plastic tubings in the flow system, where each is controlled for a different $\mathrm{pO}_{2}$ value (set values are 150 and $10 \mathrm{mmHg}$ ). The ultrasound transducer was scanned vertically in a range of $10 \mathrm{~mm}$. The step size of the scan was $0.2 \mathrm{~mm}$. At each step a sequence of PA signal acquisitions was conducted. First a background signal with only the pump laser operating was recorded. Then a series of signals was recorded at a range of pump-probe delay values. Raw PA signals were processed by first subtracting the pump-only (background) signal, and then applying a low-pass filter (eighth-order Chebishev I, cut off at $6.25 \mathrm{MHz}$ ) and envelope detection by the Hilbert transform method. A complete set of processed scanned signals is then combined to yield a PA image. The process is repeated for each value of pump-probe delay. A sample of a PA image at a pump-probe delay of $1 \mu \mathrm{s}$ is shown in Fig. 3 (bottom). The image depicts mostly the front and back interfaces of each tube. The annotated circles on the image represent the actual location and size of the inner part of the tubes. A total of $5 \mathrm{PA}$ images were calculated in the same way, corresponding to pump-probe delays of $1,3,6,10$, 


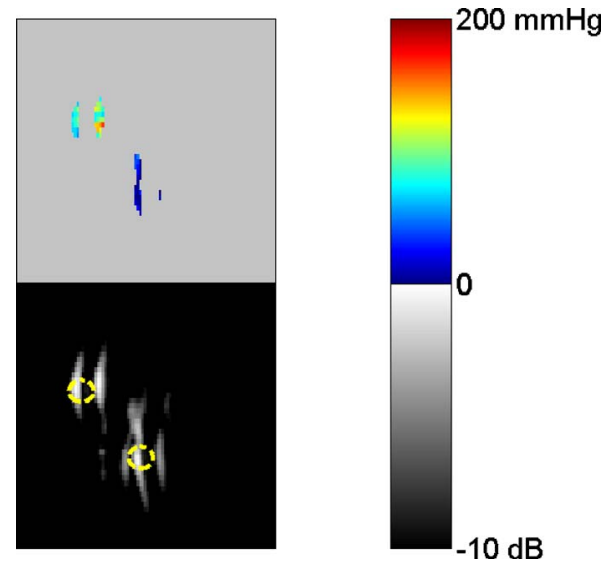

Fig. 3 (Top) PALI image of the two-tubing phantom. Color code indicates oxygen partial pressure in $\mathrm{mmHg}$. Areas where $\mathrm{pO}_{2}$ cannot be resolved due to low photoacoustic signal are represented by light gray. (Bottom) Photoacoustic amplitude image in decibel grayscale. Front and back sides of each tube appear as a double spot in the image (true location of tubes indicated as yellow dashed circle). (Color online only.)

and $20 \mu$ s. For each pixel in the images (excluding areas of low amplitude signals), a fit-to-exponential decay was performed using the five pixel values of all PA images. The decay rate was evaluated at each pixel and converted to a $\mathrm{pO}_{2}$ value using the calibration relation obtained before (Fig. 2). A colorcoded $\mathrm{pO}_{2}$ image is shown in Fig. 3 (top). The mean and standard deviation $\mathrm{pO}_{2}$ values evaluated from the image at the location of the two tubings are $102 \pm 28$ and $19 \pm 17 \mathrm{mmHg}$. The corresponding set values are 150 and $10 \mathrm{mmHg}$.

In conclusion, we present preliminary experiments demonstrating a PALI technique for oxygen imaging using MB as an oxygen sensor. Our measurements show that the MB excitation lifetime can be measured by photoacoustics, and the decay rate of the excited state depends linearly on oxygen partial pressure. The technique is suitable for measuring oxygen levels within the physiological range of $\mathrm{pO}_{2}$ from 0 to $100 \mathrm{mmHg}$. A basic imaging setup was tested for imaging a simple phantom containing two tubes of different $\mathrm{pO}_{2}$. The $\mathrm{pO}_{2}$ image correctly reflects the high and low oxygen levels in the tubes; however, significant deviations from the actual values and large scatter is encountered in the quantitative $\mathrm{pO}_{2}$ image. These errors can be attributed to fluctuations in the pulse energy of both the pump and probe lasers. A correction mechanism for normalizing the measured signals by the actual pulse energy will be considered for improving the image accuracy.
Translating this method to clinical applications in cancer would be extremely valuable for accurate prognosis and therapy planning. However, applying this method in a biological environment requires further investigations. A competing process of triplet quenching by electron transfer and radical formation can result in overestimation of $\mathrm{pO}_{2}$. The relative contribution of this process increases in low $\mathrm{pH}$ environments. ${ }^{11}$ These effects should be addressed in future testing and development of the PALI technique.

\section{References}

1. J. M. Arbeit, J. M. Brown, K. S. C. Chao, J. D. Chapman, W. C. Eckelman, A. W. Fyles, A. J. Giaccia, R. P. Hill, C. J. Koch, M. C. Krishna, K. A. Krohn, J. S. Lewis, R. P. Mason, G. Melillo, A. R. Padhani, G. Powis, J. G. Rajendran, R. Reba, S. P. Robinson, G. L. Semenza, H. M. Swartz, P. Vaupel, and D. Yang, "Hypoxia: Importance in tumor biology, noninvasive measurement by imaging, and value of its measurement in the management of cancer therapy," Int. J. Radiat. Biol. 82(10), 699-757 (2006).

2. J. W. Kim, P. Gao, and C. V. Dang, "Effects of hypoxia on tumor metabolism," Cancer Metastasis Rev. 26(2), 291-298 (2007).

3. L. N. Wang, J. C. Georgi, M. Narayanan, J. Guillem, H. Schoder, and J. L. Humm, "Pharmacokinetic analysis of hypoxia (18)Ffluoromisonidazole dynamic PET in head and neck cancer," J. Nucl. Med. 51(1), 37-45 (2010).

4. U. Wedegartner, H. Kooijman, T. Andreas, N. Beindorff, K. Hecher, and G. Adam, "T2 and T2* measurements of fetal brain oxygenation during hypoxia with MRI at 3T: correlation with fetal arterial blood oxygen saturation," Eur. Radiol. 20(1), 121-127 (2010).

5. I. Dunphy, S. A. Vinogradov, and D. F. Wilson, "Oxyphor R2 and G2: phosphors for measuring oxygen by oxygen-dependent quenching of phosphorescence," Anal. Biochem. 310(2), 191-198 (2002).

6. S. Ashkenazi, S. W. Huang, T. Horvath, Y. E. L. Koo, and R. Kopelman, "Photoacoustic probing of fluorophore excited state lifetime with application to oxygen sensing," J. Biomed. Opt. 13(3), 034023 (2008).

7. M. Gonzalez-Bejar, P. Montes-Navajas, H. Garcia, and J. C. Scaiano, "Methylene blue encapsulation in cucurbit[7]uril: laser flash photolysis and near-IR luminescence studies of the interaction with oxygen," Langmuir 25(18), 10490-10494 (2009).

8. K. Orth, A. Ruck, G. Beck, A. Stanescu, and H. G. Beger, "Photodynamic therapy of small adenocarcinomas with methylene blue," Chirurg 66(12), 1254-1257 (1995).

9. Y. Lu, R. Q. Jiao, X. P. Chen, J. Y. Zhong, A. G. Ji, and P. P. Shen, "Methylene blue-mediated photodynamic therapy induces mitochondria-dependent apoptosis in HeLa cell," J. Cell. Biochem. 105(6), 1451-1460 (2008).

10. F. Aghahosseini, F. Arbabi-Kalati, L. A. Fashtami, G. E. Djavid, M. Fateh, and J. M. Beitollahi, "Methylene blue-mediated photodynamic therapy: A possible alternative treatment for oral lichen planus," $\mathrm{La}$ sers Surg. Med. 38(1), 33-38 (2006).

11. T. João Paulo, G. Auro Del, O. Carla Santos de, G. Dino Santesso, J. Helena Couto, T. Dayane Batista, S. Divinomar, T. Rozane de Fátima, and S. B. Mauricio, "Methylene blue in photodynamic therapy: From basic mechanisms to clinical applications," Photodiagnosis and Photodynamic Therapy 2(3), 175-191 (2005).

12. M. H. Xu and L. H. V. Wang, "Photoacoustic imaging in biomedicine," Rev. Sci. Instrum. 77(4), 041101 (2006). 